\title{
Magnetic Shielding and Vacuum Test for Passive Hydrogen Masers
}

\author{
D.U. Gubser, S.A. Wolf, A.B. Jacoby, and L.D. Jones \\ Naval Research Laboratory \\ Washington, DC 20375
}

\begin{abstract}
Vibration tests on high permeability magnetic shields used in the SAO-NRL Advanced Development Model (ADM) hydrogen maser have been made. Magnetic shielding factors were measured before and after vibration at the Goddard Space Flight Center, magnetic field facility. Preliminary results indicate considerable $(<25 \%)$ degradation.

Test results on the NRL designed vacuum pumping station for the ADM hydrogen maser are al so discussed. This system employs sintered zirconium carbon getter pumps, supplied by SAES Getters, to pump hydrogen plus small ion pumps to pump the inert gases. In situ activation tests and pumping characteristics indicate that the system can meet design specifications.
\end{abstract}

\section{INTRODUCTION}

Hydrogen masers to be used as clocks aboard navigation satellites must be light but rugged enough to withstand shock and vibration introduced during launch. For the past several years, we have been developing both the magnetic shielding system and the vacuum pumping system for such a hydrogen maser. In part I of this two part article we report on preliminary shock and vibration tests performed on magnetic shields designed for a space-worthy hydrogen maser. In part II, we report on vacuum and pumping characteristics of our small, lightweight hydrogen maser pumping system.

\section{MAGNETIC SHIELDS}

A compact, lightweight magnetic shielding system using high permeability material has been designed, and tested by us and previously

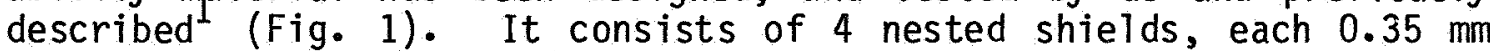
thick, with an OD of $19.4 \mathrm{~cm}$, and ID of $13.8 \mathrm{~cm}$, and overall Tength of $30 \mathrm{~cm}$. In order to test the vulnerability of these shields to shock and vibration they were assembled within a special harness in a manner which duplicated as close as possible the actual hydrogen maser mounting arrangement including heaters, thermal blankets and a "dummy" Toad 
replicating the mass of the maser cavity. At various locations on each individual shield, accel erometers were mounted to measure the magnitude of the vibration and to test for unwanted system resonances. The shields were vibrated at NRL's shock and vibration laboratory in a manner which simulated expected launch levels. Figure 2 shows the vibration table with the instrumented shielding system attached.

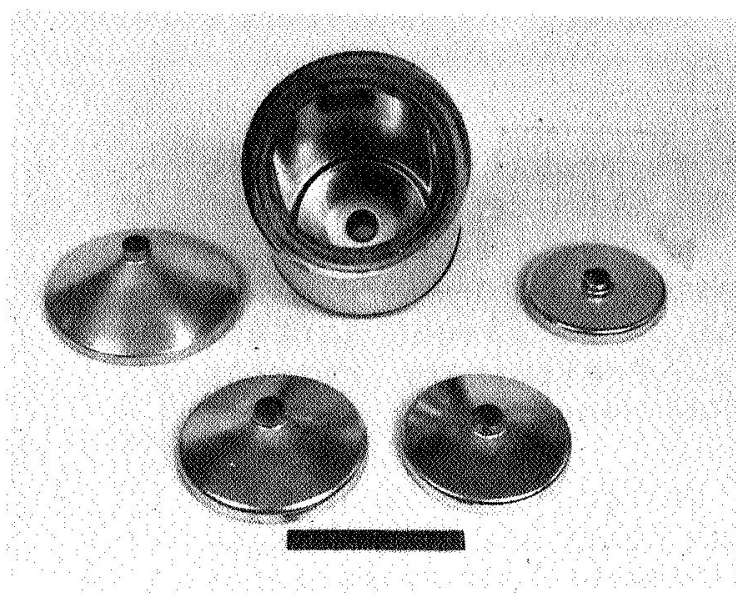

Fig. 1. Photo of Magnetic Shield system for hydrogen Maser.

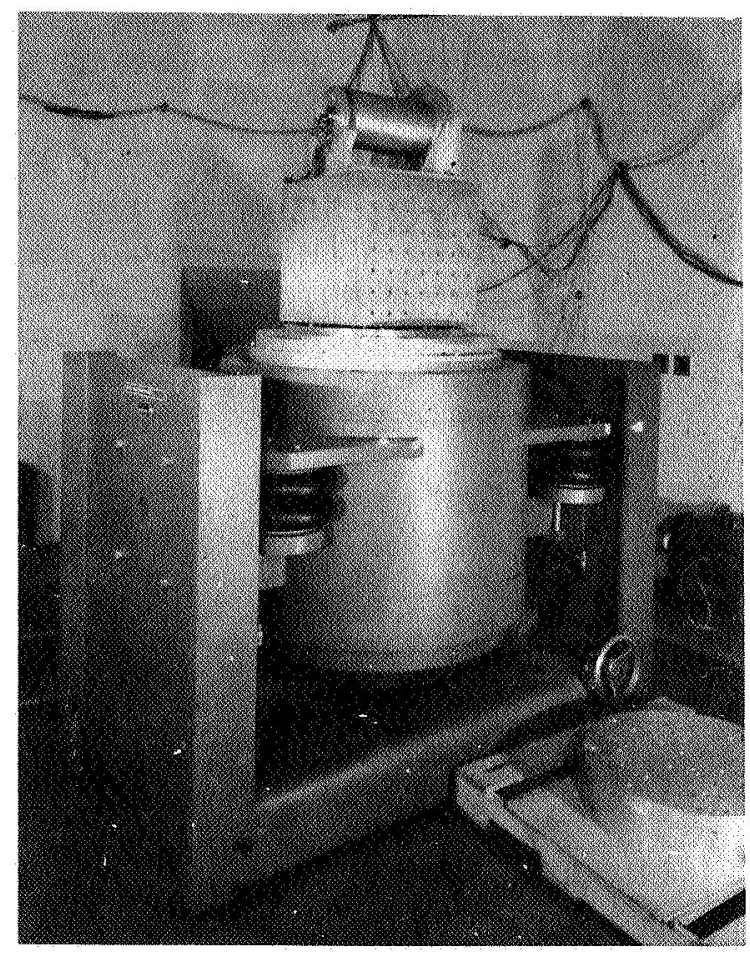

Fig. 2

Photo of Assembled Shield system showing instrumented mounting harness atop NRL vibrating table.

The magnetic shielding factor was measured before and after vibiration at the Goddard Space Fi ight Center where homogeneous fields of $\pm 60 \mu \mathrm{T}$ could be externally applied. Internal fields were measured with a flux gate magnetometer with. \pm InT resolution. The shielding factor $\Delta \mathrm{H}_{\text {ext }} /$ $\Delta \mathrm{H}_{\text {int }}$ before vibration testing was 55,000 . This value is $50 \%$ lower than previous shields of similar design and indicates that fabrication strains in the high permeability material were not completely removed during the factory annealling procedure.

Figure 3 is a plot of the power spectral density of vibration as a function of frequency. Curve " $a$ " is from a "control" accelerometer 
mounted on the harness of the system during vibration tests. Curve " $b$ " is the power spectra density from an accelerometer mounted on one of the shields during the same test. As can be seen there is a considerable difference in the two curves. The shield at the location of the accelerometer is experiencing significant, frequency dependent resonances indicating shield flexing during vibration. Spectra from accelerometers located at other spots on the shields varied in detail, but all showed frequency dependent resonances.
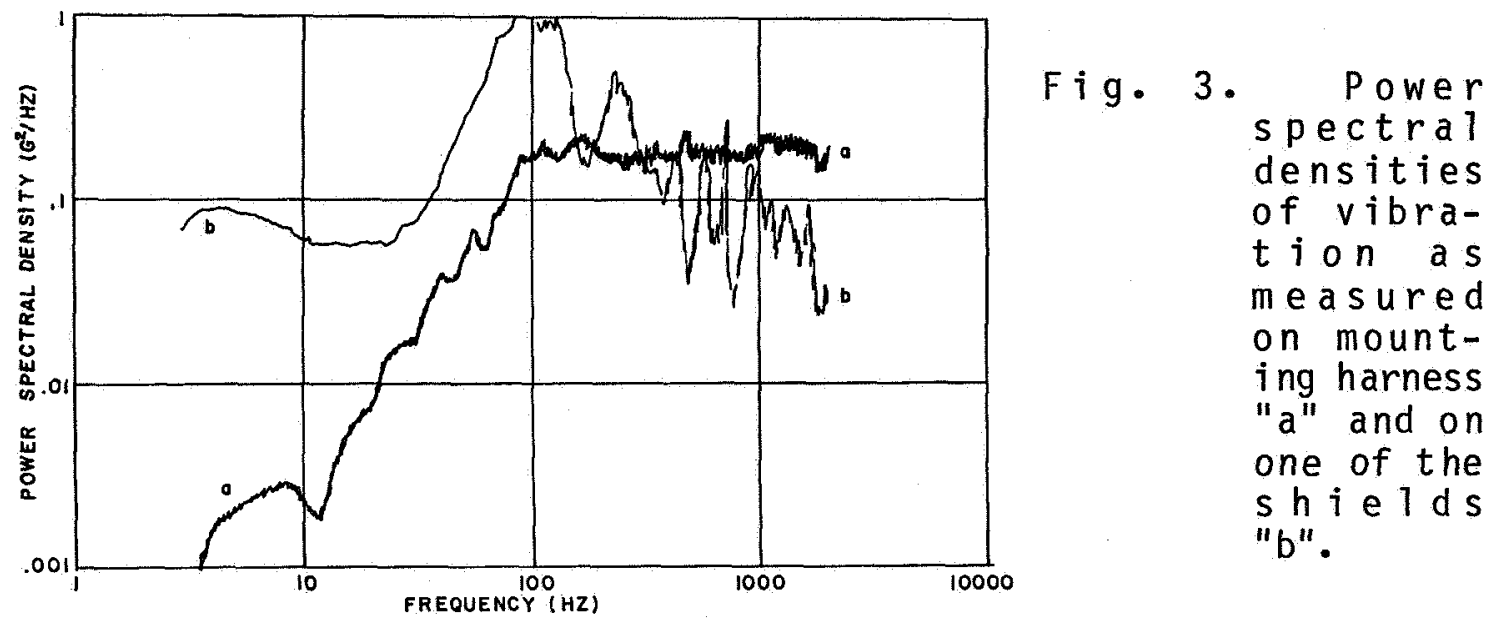

Following the vibration tests, the shielding factor was again measured and found to be reduced from 55,000 to 40,000 - a degradation of $25 \%$ ! This amount of degradation is quite large. It is possible that the initial strains in this poorly annealed set of shields may have predispositioned the shields to a more rapid degradation. We will soon be testing a more carefully annealed shield set in order to ascertain the degradation level in a shielding system more likely to be employed in an operating maser.

Different mounting arrangements for the magnetic shields may alleviate some of the problems observed with vibration induced shielding degradation. More shock absorbing or cushioning material between the shields may prevent some of the resonances. Alternatively one could increase the thickness of the shields which will give them more rigidity while at the same time provide a greater initial shielding factor for safety margin against degradation. Increasing the shield thickness by $20 \%$ will double the shielding factor for a 4-shield system, i.e. changing the shield thickness from .35 to $.43 \mathrm{~mm}$ should provide an adequate safety margin even using the present mounting arrangement.

\section{SUMMARY/CONCLUSION}

We have demonstrated a potential shock and vibration problem associated with the magnetic shield system of a space-borne hydrogen maser. 
Shielding factor reduction of $25 \%$ have been seen on a poorly annealed shield set. Future tests on better annealed shields and different mounting arrangements should suggest ways to alleviate some of the problem.

\section{VACUUM/PUMPING SYSTEM}

The vacuum/pumping system was designed to be compact, lightweight and structurally strong. A complete review of the various design considerations was presented last year. 2 The system as finally constructed is schematically shown in Fig. 4. It consists of a Ti-6Al/4V chamber which houses the state selector magnet and 2 ST $171 \mathrm{Zr}-\mathrm{C}$ getter pumps built by SAES Getters. Attached to one port on this chamber were 2 small Varian appendage ion pumps $\left(\frac{1}{2} l / s\right)$ for assisting the getter pumps with residual gases (other than $\mathrm{H}_{2}$ ). This chamber was welded to the hydrogen maser and the hydrogen disassociator was attached with a viton "0"-ring to the bottom flange. The vacuum/pumping system tests were designed to determine i) the activation procedure ii) the system pumping speed and total hydrogen capacity of the getter pumps and iij) residual gas contamination levels and operation of small appendage ion pumps. The maser was initially operated using the described pumping system but with a turbo-molecular pump instead of the getter pumps in order to ascertain the initial operating condition of the complete system prior to the in situ getter pump tests.

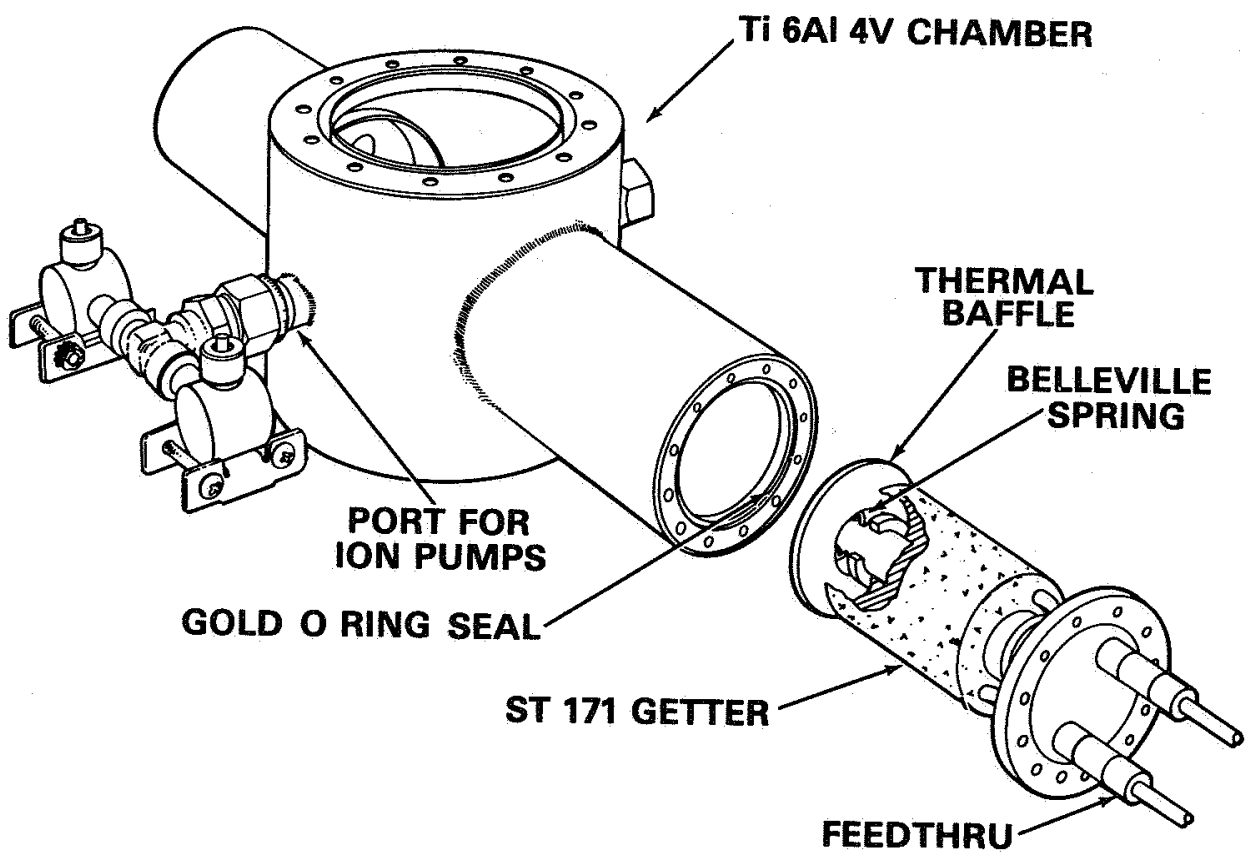

Fig. 4. Schematic drawing of Lightweight pumping system for $\mathrm{H}_{2}$ Maser. 
We used only $1 \mathrm{Zr}-\mathrm{C}$ getter pump in the performance tests. The second getter pump port was used to attach a turbo-molecular pump for system evacuation during activation and for insertion of thermocouple (TC) leads into the vacuum space. One TC was attached to the getter pump to monitor its temperature while the 2 nd was attached to the state selector magnet (AINiCo VII, hexapole) immediately facing the getter pump. The two appendage ion pumps were attached to one of the remaining small ports while a partial pressure analyzer (Perkin-ELMER/UTtek Inc. Model 607-1000) was attached to the remaining port. Thus we were able to monitor partial pressures of all gases present in the system at all times.

\section{i) Activation Tests}

The getter pumps where equipped with an internal heater for activation. Initial attempts to activate the pumps were thwarted by heater burn out and shorts (a manufacturing problem hopefully being corrected) and by a large thermal leakage from the pumps to the outside world. A design modification involving insertion of several internal radiation shields located 1) inside the Ti pumping arm surrounding the getter pump, 2) between the inner diameter of the getter pump and the central support rod and 3 ) between the feed thru flange and the face of the pump. These design changes reduced the power required to activate the pumps by $75 \%$ ! During activation, the Ti arm housing the getter pump was water cooled on the outside by slipping a double "0"-ring sealed brass cooling collar over it. The cooling water was thus directly in contact with the Ti metal itself. Activation was accomplished by maintaining the getter material at $900^{\circ} \mathrm{C}$ for 30 minutes as recommended by SAES Getters. 475 watts of power ( 8.5 amps at 55 volts ac) were required for activation. During activation the state selector magnet reached a maximum temperature of $195^{\circ} \mathrm{C}$ - well bel ow the range where degradiation is anticipated The vacuum was maintained in the $10^{-5}$ torr range or below with the turbomolecular pump. Predominate gases emitted during activation were $\mathrm{H}_{2} \mathrm{O}$, $\mathrm{CO}, \mathrm{N}_{2}$, and hydrocarbons. After activation, the system was allowed to cool, the turbo-molecular pump was valved off, and an ultimate pressure of $4 \times 10^{-7}$ torr was attained with the two ion appendage pumps and the getter pump. (See Table I)

\section{ii) Pumping Speed and Capacity}

Initial tests of the pumping speed were attempted using a variable leak valve (Granville-Phillips, Model 203) attached to a container of 5-9 purity Hydrogen. Even this high purity gas had enough $\mathrm{Ar}$ and $\mathrm{N}$ impurities to vitiate a long term test. The ion pumps could not maintain low $A r$ and $N$ levels at the accelerated flow rate desired for the test. Following this discovery, a $\mathrm{Pd}$ value was inserted in the $\mathrm{H}_{2}$ gas stream in order to further cleanse the gas. 
After insertion of the Pd value in the $\mathrm{H}_{2}$ flow, the entering gas was free from any detectable impurities except a small amount of He. The pumping speed was determined by filling an external container of known volume $V_{\text {ext }}\left(8.3\right.$ liters) with 800 Torr $\mathrm{H}_{2}$ and monitoring the pressure drop with a Quartz Manometer as the gas flowed into the maser, $\mathrm{dP}_{\text {ext }} / \mathrm{dt}$. The pressure inside the maser $\mathrm{P}_{\text {int }}$ was measured with the PPA (total pressure) and the flow rate was calculated from the formula

$$
S(l / \text { sec })=\frac{V_{\text {ext }}}{P_{\text {int }}} \frac{d P_{\text {ext }}}{d t} .
$$

The measured average pumping speed was 20 liters/sec during the 1st 30 days of the test. Since the reported pumping speed of the Getter pump is $>100 \mathrm{l} / \mathrm{sec}$, we assume that $20 \mathrm{l} / \mathrm{sec}$ represents a conduction limited speed dependent on the geometry of the baffles and support mechanisms of the getter pumps. The flow rate for this test was $10^{-4}$ torr $\ell / \mathrm{sec}$ which is approximately 5 times larger than during maser operation. The total capacity of the $\mathrm{H}_{2}$ pumped as of the writing of this article is 400 torr liters - $1 / 5$ the expected useful capacity. These numbers are listed in Table I.

\section{iii Residual Gas Contamination - ion pump operation}

A primary concern in these tests was the residual gas levels $\left(\neq \mathrm{H}_{2}\right)$ in the system which might contaminate the getter pumps, limiting theif useful lifetime and capacity. Associated with this same concern was the operation of the small ion pumps which were incorporated into the system to alleviate these residue gas problems.

For a clean system, calculations suggested that two small Varian appendage pumps would suffice to maintain stable maser operation provided the ion pumps were not degraded with time due to operation in a high partial pressure of $\mathrm{H}_{2}$. To increase the lifetime of the ion pumps operating in a hydrogen atmosphere, the operating voltage was reduced. The pumping efficiencies for lighter elements (such as $\mathrm{H}_{2}$ ) is reduced to a much greater extent than the heavier el ements (such as $\mathrm{N}, 0$, Ar, and hydrocarbons) as the operating voltage of the pumps is lowered. To determine the optimum operating voltage, we measured the residual gas levels in the system as a function of ion pump voltage for two conditions: 1) $\mathrm{H}_{2}$ flow with no Pd value (Fig. 5a) and 2) $\mathrm{H}_{2}$ flow with Pd value (Fig. $\left.5 b^{6}\right)$.

In Fig. 5a one notes substantial levels of $\mathrm{Ar}$ and $\mathrm{N}_{2}$ which were in the $\mathrm{H}_{2}$ gas itself. With the ion pump off, the Ar and $\mathrm{N}$ Tevels quickly rose ahd the total pressure of the system increased continuously, i.e. the getter pumps could not pump these gases sufficiently well to maintain a 

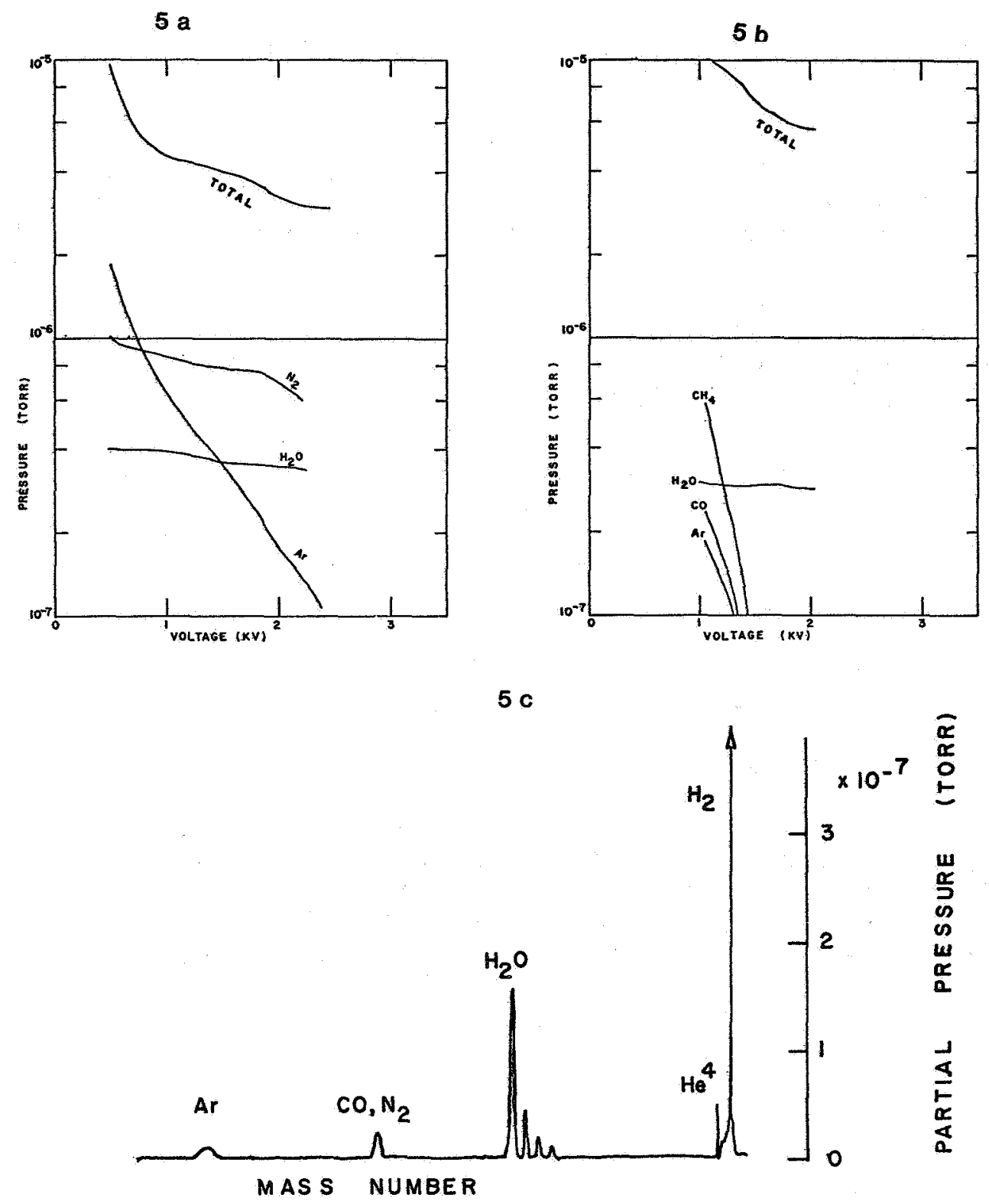

Fig. 5. Residual gas levels in $\mathrm{H}_{2}$ Maser. (See discussion Ref. 3 ). (5a) $\mathrm{H}_{2}$ flow with no Pd value

(5b) $\mathrm{H}_{2}$ flow with $\mathrm{Pd}$ value

(5c) Gas spectrum after 40 days of operation at a flow rate of $10^{-4}$ torr $\& / \mathrm{sec}$. 
constant total system pressure at the flow rate used. With an operating voltage of 1 kvolt, the system would maintain a stable pressure (albeit at a low flow rate), due to the ion pumps assistance with pumping. Ar partial pressure remained relatively high since the getters do not pump Ar. It appears that low voltage levels $1<V<2$ Kvolts can effectively pump most contaiminants in the maser. $\mathrm{H}_{2}$ does not appear to be significantly pumped at these voltage levels.

The same test was again performed after insertion of the Pd valve. Figure $5 b$ shows the gas levels vs ion voltage for this test. As $c$ an be seen, the system is much cleaner, $i . e$. the $P d$ value removed the contaminants in the $\mathrm{H}_{2}$ gas. At $1 \mathrm{Kvolt}, \mathrm{CH}_{4}, \mathrm{CO}, \mathrm{H}_{2} \mathrm{O}$ and some $\mathrm{Ar}$ was detected in the $10^{-7}$ torr range. ${ }^{3}$ At 1.5 Kvolt, all gas contamination levels had fallen into the $10^{-8}$ range except water. Thus it appears, that one can maintain a clean system operating the Varian appendage pumps at voltages as low as 1.5 Kvolts. We chose to operate the pumps at 1.75 Kvolt for the lifetime tests which are in progress. A PPA trace showing the detectable gas levels of the system after operation for 40 days is shown in Fig. $5 \mathrm{c}$. Note the $\mathrm{H}_{2} \mathrm{O}$ peak is $50 \%$ lower after 40 days than it was when the data in Fig. $5 \$$ was obtained after 5 days of operation, $i . e$. the system is cleaning up with time.

\section{SUMMARY/CONCLUS IONS}

We have now demonstrated that the major design features of the lightweight Vacuum Pumping System are attainable. Major problems/uncertainties remaining are associated with 1) manufacturers reliability for delivering quality getter pumps with internal heater and 2) long lifetime tests for both the getter pumps and the ion pumps. 


\section{TABLE I}

Pump System Characteristics

Activation power
Activation temperature
Activation time
UTtimate vacuum
Pumping speed

System pressure

Total pumped capacity at time of article

Ion Pump Power
475 watts

$900^{\circ} \mathrm{C}$

30 minutes

$\begin{array}{ll}4 \times 10^{-7} & \text { getter }+ \text { ion } \\ 9 \times 10^{-7} & \text { getter }\end{array}$

$20 / \mathrm{sec}$

$5.5 \times 10^{-6}$ torr (at flow rate of $10^{-4}$ torr $\ell / \mathrm{sec}$ )

(40 days) 400 torr liters

$<1$ watt per pump

\section{REFERENCES}

1. D.U. Gubser, S.A. Wolf, and J.E. Cox, Rev. Sci. Instru. 56 , 751 (1979).

2. S.A. Wolf, D.U. Gubser, and L.D. Jones, Proceedings of the 12th Annual PTTI meeting.

3. The partial pressures measured and reported are only nominal. The Uitek PPA does not accurately determine partial pressures of residual gasses, but rather gives a signal approximately proportional to their relative abundance in the system. The total pressure readings, however, are quite accurate since in this mode the PPA operates as an ionization guage. 